\title{
The Stochastic Geometric Machine Model ${ }^{1}$
}

R.H.S. REISER, G.P. DIMURO, A.C.R. COSTA², Escola de Informática, Universidade Católica de Pelotas, 96010-000 Pelotas, RS, Brazil3.

\begin{abstract}
This paper introduces the stochastic version of the Geometric Machine Model for the modelling of sequential, alternative, parallel (synchronous) and nondeterministic computations with stochastic numbers stored in a (possibly infinite) shared memory. The programming language $\mathcal{L}\left(\mathbb{D}_{\infty}\right)$, induced by the Coherence Space of Processes $\mathbb{D}_{\infty}$, can be applied to sequential and parallel products in order to provide recursive definitions for such processes, together with a domain-theoretic semantics of the Stochastic Arithmetic. We analyze both the spacial (ordinal) recursion, related to spacial modelling of the stochastic memory, and the temporal (structural) recursion, given by the inclusion relation modelling partial objects in the ordered structure of process construction.
\end{abstract}

\section{Introduction}

Following the same methodology used for the construction of the Interval Geometric Machine [8], in this paper we introduce the stochastic version of the Geometric Machine Model [6], applying the computational approach suggested by Scott [9]. Based on Domain Theory [10], the Girard's domains called Coherence Spaces [2] characterize the ordered structure of the Stochastic Geometric Machine (SGM). Coherent subsets are conceived as linear traces of linear functions interpreting states and processes of the SGM. The notion of coherence between coherent subsets provides semantic interpretation for parallel and non-deterministic computations performed over array structure of stochastic numbers [4], represented by pairs of elements of the subspace of total objects of the Bi-structured Coherence Space of Rational Intervals, $\mathbb{I} \mathbb{Q}[1]$, a constructive computational representation of the set of real numbers. The non-deterministic machine states are conceived as collections of linear functions (with singletons modelling deterministic states) from the coherence space $\mathbb{R}^{3}$, interpreting the memory positions related to the euclidian geometric space, to the coherence space of values $(\mathbb{I} \mathbb{Q})$, modelling both the set of mean values and the set of standard deviations of stochastic numbers. Over the inductive ordered structure called the Coherence Space of Processes $\left(\mathbb{D}_{\infty}\right)$, parallel and non-deterministic processes operating on the array structures of the SGM memory are modelled. A process, interpreted in the the domain $\mathbb{D}_{\infty}$ as a coherent subset of linear functions, can be represented as an expression in the textual language $\mathcal{L}\left(\mathbb{D}_{\infty}\right)$ [7]. A stochastic

\footnotetext{
${ }^{1}$ This work was partially supported by CTINFO/CNPq and FAPERGS. ${ }^{2}$ PPGC/UFRGS

${ }^{3}$ e-mail: $\{$ reiser, dimuro, costa\}@atlas.ucpel.tche.br
} 
number is identified by a pair $(i, j)$ of memory positions, with expressions $s[i, j, 0]$ and $s[i, j, 1]$ representing its value and its deviation, respectively. A connection between the textual language and the domain-theoretic structure of the algorithms involving the arithmetic of stochastic numbers is presented.

\section{Stochastic Numbers and Stochastic Arithmetic}

Most of the algebraic properties of the exact arithmetic can be recovered in stochastic arithmetic because it takes into consideration the numerical instabilities during the run of a program. The use of stochastic arithmetic in computing makes possible to estimate the accuracy of any result provided by a computer. In the fields of roundoff error analysis and validity of numerical software, it makes possible to develop theoretical studies. In [4], an algebraic approach abstracted from the properties

of stochastic arithmetic permits to reduce computation with stochastic numbers to computation in familiar vector spaces. For that, a stochastic number is conceived as a gaussian random variable (in the continuous space) with a known mean value (real number) and a known standard deviation (nonnegative real number). This notion of (proper, usual) deviation is generalized and improper (negative) standard deviations are studied. The algebraic properties of vector spaces are induced by s-spaces (with group structure) defined over the set $\mathrm{R}$ of real numbers leading to a generalization of the familiar concepts of linear dependence and basis and provides an algebraic structure analogous to quasilinear spaces, which appears in interval analysis [3]. In the following we resume the main aspects of stochastic arithmetic.

Definition 2.1. A stochastic number $X$ is a gaussian random variable with known mean value $m$ and standard deviation $s$, i.e., $\mathcal{S}=\left\{X=(m ; s) \mid m \in \mathrm{R}, s \in \mathrm{R}^{+}\right\}$.

Let $X_{1}=\left(m_{1} ; s_{1}\right), X_{2}=\left(m_{2} ; s_{2}\right) \in \mathcal{S}$. Equality between two stochastic numbers $X_{1}, X_{2}$ is given by $X_{1}=X_{2}$ if $m_{1}=m_{2}$ and $s_{1}=s_{2}$.

Definition 2.2. The addition and scalar multiplication is defined by:

$$
\begin{aligned}
X_{1}+X_{2} & =\left(m_{1} ; s_{1}\right)+\left(m_{1} ; s_{1}\right)=\left(m_{1}+m_{2} ; \sqrt{s_{1}^{2}+s_{2}^{2}}\right), \\
\alpha * X_{1} & =\alpha *\left(m_{1} ; s_{1}\right)=\left(\alpha m_{1},|\alpha| s_{1}\right) .
\end{aligned}
$$

A stochastic number $(0 ; s)$ is called symmetric (with respect to the origin). The set of symmetric stochastic numbers is denoted by $\mathcal{S}_{+}=\left\{(0 ; s) \mid s \in \mathrm{R}_{+}\right\}$. If $X_{1}, X_{2} \in \mathcal{S}_{+}$, then $\forall \alpha \in \mathrm{R}, X_{1}+X_{2} \in \mathcal{S}_{+}$. In [4], it is shown that $\left(\mathcal{S}_{+},+, \mathbb{R}, *\right)$ is an s-space of monoid structure. Thus, for $\alpha, \beta \in \mathbb{R}, X, X_{1}, X_{2} \in \mathbb{R}_{+}$, it holds that

$$
\begin{array}{rlrl}
\alpha *\left(X_{1}+X_{2}\right) & \left.=\alpha * X_{1}+\alpha * X_{2}\right) ; & & 1 * X=X ; \\
\alpha *(\beta * X) & =(\alpha \beta) * X ; & & (-1) * X=X ; \\
\sqrt{\alpha^{2}+\beta^{2}} * X & =\alpha * X+\beta * X, \alpha, \beta \geq 0 .
\end{array}
$$

In (2.5) it must hold $\alpha, \beta \geq 0$ and thus, this property is not convenient for algebraic computations. An unconditional relation is considered now. 
A stochastic number of the form $(0 ; s)$, with $s \in \mathbb{R}$, is called extended symmetric. In this case, $s$ belongs to the set $\mathcal{S}_{\oplus}=\{(0 ; s) \mid s \in \mathrm{R}\}$ of extended symmetric stochastic numbers. To obtain the extended symmetric stochastic arithmetic, we consider the signed square root $\sqrt{\alpha}^{\circ}=\sigma(\alpha) \sqrt{|\alpha|}$, whenever $\alpha \in \mathbb{R}$ and $\sigma(\alpha)=+$ if $\alpha \geq 0$ or $\sigma(\alpha)=-$, otherwise.

Definition 2.3. Let $X_{1}, X_{2} \in \mathcal{S}_{\oplus} . X_{1} \oplus X_{2} \in \mathcal{S}_{\oplus}$ is defined by

$$
X_{1} \oplus X_{2}=\left(0 ; \sqrt{\sigma\left(s_{1}\right) s_{1}^{2}+\sigma\left(s_{2}\right) s_{2}^{2}}\right) .
$$

The structured set $(\mathcal{S}, \oplus, \mathbb{R}, *)$ is an s-space over $\mathbb{R}$ with group structure, where $X \oplus o p p(X)=\mathbf{0}$ whenever $\mathbf{0}=(0 ; 0)$ and $o p p(X)=o p p(0 ; s)=(0,-s)$. In addition, if $X_{+}=X$ and $X_{-}=o p p(X)$, for $\alpha, \beta \in \mathbb{R}, X \in \mathcal{S}_{\oplus}$, it holds that

$$
{\sqrt{\alpha^{2}+\beta^{2}}}^{\circ} * X_{\sigma(\alpha+\beta)}=\sqrt{\alpha}^{\circ} * X_{\sigma(\alpha)} \oplus \sqrt{\beta}^{\circ} * X_{\sigma(\beta)} .
$$

\section{The ordered structure of the SGM}

The ordered structure of the SGM is inspired on the ordered structure of the interval version of the Geometric Machine Model [7, 8]. The methodology of construction is developed by levels, starting from the coherence space $\mathbb{D}_{0}$ of elementary processes. The intuitive notion of an elementary process, which modifies a single memory position in a single unit of computational time (uct), can be described by an elementary transition between deterministic memory states, given by a linear function. The next coherence space in the construction, denoted by $\overline{\mathbb{D}}_{0}$, models concurrent sets of elementary processes. The coherence relation between such processes models the admissibility of parallelism between them and essentially says that two elementary processes can be performed in parallel if they do not conflict, i.e., if they do not access the same memory position. That relation defines also the web over which the coherence space of the whole set of processes in the model is step-wise and systematically build. The domain $\overline{\mathbb{D}}_{0}$ is thus the domain of parallel products of elementary processes. In the dual construction, $\overline{\mathbb{D}}_{0}^{\perp}$, justified by the presence of involutive negation $\perp$ of coherence spaces [2], the incoherence relation models the condition for non-determinism, namely, the conflict of memory accesses. For the construction of the sequential product and the deterministic sum, consider $\mathbb{P}_{0} \equiv \mathbb{D}_{0} \bigsqcup \overline{\mathbb{D}}_{0} \bigsqcup \overline{\mathbb{D}}_{0}^{\perp}$ as the amalgamated (smash) sum of $\mathbb{D}_{0}, \overline{\mathbb{D}}_{0}$ and $\overline{\mathbb{D}}_{0}^{\perp}$. The direct product $\mathbb{P}_{0} \prod \mathbb{P}_{0}$ is the coherence space of sequential products of two (parallel, non-deterministic or elementary) processes, whose execution is performed in 2 uct. The coherence space $\mathbb{P}_{0} \prod_{\mathbb{B}} \mathbb{P}_{0}$ of the deterministic sums of (parallel, nondeterministic or elementary) processes, performed in 2 uct, is defined as the direct product between $\mathbb{B}$ and $\mathbb{P}_{0} \prod \mathbb{P}_{0}$. The coherence space $\mathbb{D}_{1}$, given by

$$
\mathbb{D}_{1}=\mathbb{P}_{0} \amalg\left(\mathbb{P}_{0} \prod \mathbb{P}_{0}\right) \amalg\left(\mathbb{P}_{0} \prod_{\mathbb{B}} \mathbb{P}_{0}\right) \text { where } \mathbb{P}_{0}=\mathbb{D}_{0} \amalg \overline{\mathbb{D}}_{0} \amalg \overline{\mathbb{D}}_{0}^{\perp},
$$

encompasses the first level of the construction of the ordered structure of the GM model and provides the representations for all processes performed in at most $2 u c t$.

Generalizing these ideas, each level identifies a subspace $\mathbb{D}_{n+1}$, 


$$
\mathbb{D}_{n+1}=\mathbb{P}_{n} \amalg\left(\mathbb{P}_{n} \prod \mathbb{P}_{n}\right) \amalg\left(\mathbb{P}_{n} \prod_{\mathbb{B}} \mathbb{P}_{n}\right), \quad \mathbb{P}_{n}=\mathbb{D}_{n} \amalg \overline{\mathbb{D}}_{n} \coprod \overline{\mathbb{D}}_{n}^{\perp},
$$

which reconstructs the objects from the level below it, preserving their properties, and constructs the new objects specific of this level. The constructive relationship between the levels is expressed by linear functions called embedding and projection functions, which model constructors and destructors of processes, respectively.

Definition 3.1. Consider the indexes $\beta \in\{0,1\}, \theta \in\{0,1,2\}$ to introduce the embedding-functions and related projections

1. (a) $\gamma_{n}^{(0)}, \gamma_{n}^{(1)}, \gamma_{n}^{(2)}: \mathbb{P}_{n}, \mathbb{P}_{n} \prod \mathbb{P}_{n}, \mathbb{P}_{n} \prod_{\mathbb{B}} \mathbb{P}_{n} \rightarrow \mathbb{D}_{n+1}, \gamma_{n}^{(\theta)}(x)=\left\{\mathbf{a}_{\theta} \mid \mathbf{a} \in x\right\} ;$

(b) $\Gamma_{n}^{(0)}, \Gamma_{n}^{(1)}, \Gamma_{n}^{(2)}: \mathbb{D}_{n+1} \rightarrow \mathbb{P}_{n}, \mathbb{P}_{n} \prod \mathbb{P}_{n}, \mathbb{P}_{n} \prod_{\mathbb{B}} \mathbb{P}_{n}, \Gamma_{n}^{(\theta)}(x)=\left\{\mathbf{a} \mid \mathbf{a}_{\theta} \in x\right\}$.

2. (a) $\phi_{n}^{(0)}, \phi_{n}^{(1)}, \phi_{n}^{(2)}: \mathbb{D}_{n}, \overline{\mathbb{D}}_{n}, \overline{\mathbb{D}}^{\perp} \rightarrow \mathbb{P}_{n}, \phi_{n}^{(\theta)}(x)=\left\{\mathbf{a}_{\theta} \mid \mathbf{a} \in x\right\}$;

(b) $\Phi_{n}^{(0)}, \Phi_{n}^{(1)}, \Phi_{n}^{(2)}: \mathbb{P}_{n} \rightarrow \mathbb{D}_{n}, \overline{\mathbb{D}}_{n}, \overline{\mathbb{D}}^{\perp}, \quad \Phi_{n}^{(\theta)}(x)=\left\{\mathbf{a} \mid \mathbf{a}_{\theta} \in x\right\}$.

3. (a) $\psi_{n}^{(\beta)}: \mathbb{D}_{n} \rightarrow \mathbb{P}_{n} \prod \mathbb{P}_{n}, \quad \psi_{n}^{(\beta)}(x)=\left\{\mathbf{a}_{\beta} \mid \mathbf{a} \in x\right\} ;$

(b) $\Psi_{n}^{(\beta)}: \mathbb{P}_{n} \prod \mathbb{P}_{n} \rightarrow \mathbb{D}_{n}, \quad \Psi_{n}^{(\beta)}(x)=\left\{\mathbf{a} \mid \mathbf{a}_{\beta} \in x\right\}$.

4. (a) $\lambda_{n}^{(0)}, \lambda_{n}^{(1)}, \lambda_{n}^{(2)}: \mathbb{P}_{n}, \mathbb{P}_{n}, \mathbb{B} \rightarrow \mathbb{P}_{n} \prod_{\mathbb{B}} \mathbb{P}_{n}, \quad \lambda_{n}^{(\theta)}(x)=\left\{\mathbf{a}_{\theta} \mid \mathbf{a} \in x\right\} ;$

(b) $\Lambda_{n}^{(0)}, \Lambda_{n}^{(1)}, \Lambda_{n}^{(2)}: \mathbb{P}_{n} \prod_{\mathbb{B}} \mathbb{P}_{n} \rightarrow \mathbb{P}_{n}, \mathbb{P}_{n}, \mathbb{B}, \quad \Lambda_{n}^{(\theta)}(x)=\left\{\mathbf{a} \mid \mathbf{a}_{\theta} \in x\right\}$.

Definition 3.2. Let $\theta \in\{0,1,2\}$ be an index and consider ${ }^{4}$

- $\pi_{n}^{(0)},{\pi_{n}}^{(1)}, \pi_{n}^{(2)}: \mathbb{D}_{n}, \overline{\mathbb{D}}_{n}, \overline{\mathbb{D}}_{n}^{\perp} \rightarrow \mathbb{D}_{n+1}$, and

. $\Pi_{n}^{(0)}, \Pi_{n}^{(1)}, \Pi_{n}^{(2)}: \mathbb{D}_{n+1} \rightarrow \mathbb{D}_{n}, \overline{\mathbb{D}}_{n}, \overline{\mathbb{D}}_{n}^{\perp}$

as embedding-functions and related projections, whose definitions are given by

1. if $x \in \gamma_{n}^{(0)}\left[\mathbb{P}_{n}\right], \pi_{n+1}^{\rightarrow(\theta)}(x)=\gamma_{n}^{(0)} \circ \phi_{n}^{(\theta)}, \Pi_{n+1}^{\rightarrow(\theta)}(x)=\Phi_{n}^{(\theta)} \circ \Gamma_{n}^{(0)}$;

2. if $x \in \gamma_{n}^{(1)}\left[\mathbb{P}_{n} \prod \mathbb{P}_{n}\right], \pi_{n+1}^{\rightarrow(\theta)}=\gamma_{n}^{(1)} \circ \psi_{n}^{(0)} \circ \phi_{n}^{(\theta)}, \Pi_{n+1}^{\rightarrow(\theta)}=\Phi_{n}^{(\theta)} \circ \Psi_{n}^{(0)} \circ \Gamma_{n}^{(1)}$;

3. if $x \in \gamma_{n}^{(2)}\left[\mathbb{P}_{n} \prod_{\mathbb{B}} \mathbb{P}_{n}\right], \pi_{n+1}^{\rightarrow(\theta)}(x)=\gamma_{n}^{(2)} \circ \psi_{n}^{(0)} \circ \phi_{n}^{(\theta)}, \Pi_{n+1}^{\rightarrow(\theta)}(x)=\Phi_{n}^{(\theta)} \circ \Psi_{n}^{(0)} \circ \Gamma_{n}^{(2)}$.

According Definição 3.1, 3.2, an element in the web of a domain $\mathbb{D}_{n+1}$ is indexed by two or tree symbols. The leftmost symbol of an index indicates one of the following constructors - (0) (for the simple inclusion of an element of the previous domain $\mathbb{D}_{n}$ in the new domain $\mathbb{P}_{n}$ related to the sub-level $\mathbb{D}_{n}-\mathbb{P}_{n}$ ), (1) (indicating the parallel product of elements existing in the previous domain $\mathbb{P}_{n}$ ) or (2) (indicating the non-deterministic sum of elements existing in the previous level). The second and third symbols related to the sub-level $\mathbb{P}_{n}-\mathbb{D}_{n+1}$, if present, mean the following: that the element is the first (02) or the second (12) summand in a deterministic sum represented in $\mathbb{P}_{n} \prod_{\mathbb{B}} \mathbb{P}_{n}$, or that it is the first (01) or the second (11) term in a sequential product in $\mathbb{P}_{n} \prod \mathbb{P}_{n}$. When the index is given by two symbols, the second one is the inclusion of an element in $\mathbb{P}_{n}$ to $\mathbb{D}_{n+1}$. The coherence space $\mathbb{D}_{\infty}$ is defined as the least fixed point for the equation (3.1), representing finite processes. The completion procedure guarantees the existence of the least solution to the recursive

\footnotetext{
${ }^{4}$ The notations ${\pi_{n}^{(0)}}^{\left(0 \pi_{n} \text { and } \Pi_{n}^{(0)}\right.} \equiv \Pi_{n}$ are assumed.
} 
equations defined by composition of morphisms in $\mathbb{D}_{\infty}$. Thus, this model is able to interpret processes that have no restrictions related to memory size, and it can be completed for temporally infinite sequential processes. That is,

$$
\mathbb{D}_{\infty}^{\rightarrow}=\mathbb{P}_{\infty}^{\rightarrow} \coprod\left(\mathbb{P}_{\infty}^{\rightarrow} \prod \mathbb{P}_{\infty}^{\rightarrow}\right) \coprod\left(\mathbb{P}_{\infty}^{\rightarrow} \prod_{\mathbb{B}} \mathbb{P}_{\infty}^{\rightarrow}\right), \quad \mathbb{P}_{\infty}^{\rightarrow}=\mathbb{D}_{\infty}^{\rightarrow} \coprod \overline{\mathbb{D}}_{\infty}^{\rightarrow} \coprod \overline{\mathbb{D}}_{\infty}{ }^{\perp} .
$$

whose indexed tokens of its coherent subsets are expressed by sequences of two or three symbols together with a suffix, following the denotation:

(1) $: 00 \equiv 00.00 .00 \ldots$ related to finite processes;

(2) : $001 \equiv 001.001 \ldots$ related to infinite sequential processes;

(3) $: 002 \equiv 002.002 \ldots$ related to infinite deterministic sums.

Definition 3.3. $\pi_{n, \infty}: \mathbb{D}_{n} \rightarrow \mathbb{D}_{\infty}$ is given by $\pi_{0, \infty}(x)=\left\{\mathbf{a}_{: 00} \mid \mathbf{a} \in x\right\}$, and

(a) $\pi_{n+1, \infty}^{\rightarrow}(x)=\left\{\mathbf{a}_{: 00} \mid \mathbf{a} \in x\right\}$, if $x \in \gamma_{n}^{(0)}\left[\mathbb{P}_{n}\right]$,

(b) $\pi_{n+1, \infty}^{\rightarrow}(x)=\left\{\mathbf{a}_{: 001} \mid \mathbf{a} \in x\right\}$, if $x \in \gamma_{n}^{(1)}\left[\mathbb{P}_{n} \prod \mathbb{P}_{n}\right]$,

(c) $\pi_{n+1, \infty}(x)=\left\{\mathbf{a}_{: 002} \mid \mathbf{a} \in x\right\}$, if $x \in \gamma_{n}^{(2)}\left[\mathbb{P}_{n} \prod_{\mathbb{B}} \mathbb{P}_{n}\right]$.

Now, let $x=\bigcup_{m \in \omega}^{\uparrow}\left\{x_{m} \mid x_{m} \in \pi_{m, \infty}^{\rightarrow}\left[\mathbb{D}_{m}\right]\right\}$ be a directed subset of finite approximations of $x$. The projection-function $\Pi_{\infty, n}: \mathbb{D}_{\infty} \rightarrow \mathbb{D}_{n}$ is given by

$\Pi_{\infty, n+1}(x)=\bigcup_{m \in \omega}^{\uparrow}\left\{\Pi_{m, n} \circ \Pi_{\infty, m}^{\rightarrow}\left(x_{m}\right)\right\}$ where $\Pi_{\infty, 0}^{\rightarrow}\left(x_{m}\right)=\left\{\mathbf{d}^{k} \mid \mathbf{d}_{: 00}^{k}=x_{m}\right\}$ and (a) $\Pi_{\infty, m}^{\rightarrow}\left(x_{m}\right)=\left\{\mathbf{a} \mid \mathbf{a}_{: 00} \in x_{m}\right\}$, if $x_{m} \in\left(\pi_{m, \infty}^{\rightarrow} \circ \gamma_{m-1}^{(0)}\right)\left[\mathbb{P}_{m-1}\right]$,

(b) $\Pi_{\infty, m}^{\rightarrow}\left(x_{m}\right)=\left\{\mathbf{a} \mid \mathbf{a}_{: 001} \in x_{m}\right\}$, if $x_{m} \in\left(\pi_{m, \infty}^{\rightarrow} \circ \gamma_{m-1}^{(1)}\right)\left[\mathbb{P}_{m-1} \prod \mathbb{P}_{m-1}\right]$,

(c) $\Pi_{\infty, m}^{\rightarrow}\left(x_{m}\right)=\left\{\mathbf{a} \mid \mathbf{a}_{: 002} \in x_{m}\right\}$, if $x_{m} \in\left(\pi_{m, \infty} \circ \gamma_{m-1}^{(2)}\right)\left[\mathbb{P}_{m-1} \prod_{\mathbb{B}} \mathbb{P}_{m-1}\right]$.

Proposition 3.1. The morphisms presented in Def. 3.1, 3.2, 3.3 are linear functions.

\subsection{Semantic interpretation of the parallel product}

Induced by the flat domain $\mathbb{R}^{3}$, the memory position information on the domain $\mathbb{D}_{0}$ of elementary processes can be lifted to the coherent sets of the constructed processes by the position-function $\Upsilon_{\infty}$, formally presented in [7]. Based on that, a semantic interpretation of parallel product is presented now.

Proposition 3.2. Let $x=\bigcup_{i \in \omega}^{\uparrow}\left\{x_{i} \mid x_{i} \in \pi_{n, \infty}\left[\mathbb{D}_{n}\right]\right\}, y=\bigcup_{i \in \omega}^{\uparrow}\left\{y_{i} \mid y_{i} \in \pi_{m, \infty}\left[\mathbb{D}_{m}\right]\right\}$, be objects of $\mathbb{D}_{\infty} \overrightarrow{\text { satisfying the hypotheses }}$

- $x_{n} \in \pi_{n, \infty}^{(00)}\left[\mathbb{D}_{n}\right] \subseteq \pi_{n, \infty}^{\rightarrow}\left[\mathbb{D}_{n}\right]$ and $y_{m} \in \pi_{m, \infty}^{(00)}\left[\mathbb{D}_{m}\right] \subseteq \pi_{n, \infty}^{\rightarrow}\left[\mathbb{D}_{m}\right]$;

- $\Upsilon_{\infty}(x) \bigcap \Upsilon_{\infty}(y)=\emptyset$ and $\Upsilon_{\infty}\left(x^{\prime}\right) \bigcap \Upsilon_{\infty}\left(y^{\prime}\right)=\emptyset$ (concurrent processes);

- $\Pi_{\infty, k}^{\rightarrow}(x), \Pi_{\infty, k}^{\rightarrow}(y) \in \gamma_{k-1}^{0} \circ \phi_{k-1}^{1}\left[\mathbb{D}_{k-1}\right]$, where $k=\max \{m, n\}$.

The linear function $\boldsymbol{F}_{(10)}: \mathbb{D}_{\infty} \prod \mathbb{D}_{\infty} \rightarrow \mathbb{D}_{\infty}$ is given by

$$
\boldsymbol{F}_{(10)}(x \sqcap y)=\bigcup_{n, m}^{\uparrow}\left\{\pi_{k, \infty}^{\rightarrow}\left[\Pi_{\infty, k}^{\rightarrow}\left(x_{n}\right) \cup \Pi_{\infty, k}^{\rightarrow}\left(y_{m}\right)\right]\right\}
$$

Otherwise, if such conditions do not hold, $\boldsymbol{F}_{(10)}(x \sqcap y)=\emptyset$.

Proof. Firstly, we show that the monotonicity of function $\mathbf{F}_{(10)}$ holds. Let $x, y \in \mathbb{D}_{\infty}$

such that $x=\bigcup_{n \in \omega}^{\uparrow}\left\{x_{n}\right\}$ and $y=\bigcup_{m \in \omega}^{\uparrow}\left\{y_{m}\right\}$ whenever $x_{n} \in \pi_{n, \infty}\left[\mathbb{D}_{n}\right]$ and $y_{m} \in \pi_{m, \infty}\left[\mathbb{D}_{m}\right]$. Suppose $x \sqcap y, x^{\prime} \sqcap y^{\prime} \in \mathbb{D}_{\infty}^{\rightarrow} \prod \mathbb{D}_{\infty}$ such that $x \sqcap y \subseteq x^{\prime} \sqcap y^{\prime}$. In 
this case, $x \subseteq x^{\prime}$ and $y \subseteq y^{\prime}$. (i) Let $\Pi_{\infty, k}^{\overrightarrow{ }}, \pi_{k+1, \infty}$ and $\pi_{k+1, \infty}^{(00)}$ be monotone functions satisfying the hypotheses. Since $\Pi_{\infty, k}$ and $\pi_{k+1, \infty}^{(00)}$ are monotonic functions, it follows that $\Pi_{\infty, k}^{\rightarrow}\left(x^{\prime}\right), \Pi_{\infty, k}^{\rightarrow}\left(y^{\prime}\right) \in \gamma_{k-1}^{0} \circ \phi_{k-1}^{1}\left[\mathbb{D}_{k-1}\right]$. Therefore,

$$
\begin{aligned}
& \mathbf{F}_{(10)}(x \sqcap y)=\pi_{k+1, \infty}\left[\Pi_{\infty, k}(x) \cup \Pi_{\infty, k}^{\rightarrow}(y)\right] \\
& \left.\subseteq \pi_{k+1, \infty}\left[\Pi_{\infty, k}\left(x^{\prime}\right) \cup \Pi_{\infty, k}\left(y^{\prime}\right)\right\}\right]=\mathbf{F}_{(10)}\left(x^{\prime} \sqcap y^{\prime}\right) \text {. }
\end{aligned}
$$

(ii) Otherwise, when no hypothesis holds, $\mathbf{F}_{(10)}(x \sqcap y)=\emptyset$. Thus,

(ii.1) If $x \in \pi_{n, \infty}^{\theta_{1}}\left(\mathbb{D}_{n}\right), y \in \pi_{m, \infty}^{\theta_{2}}\left(\mathbb{D}_{m}\right), \theta_{1}, \theta_{2} \neq 00$, then $x^{\prime} \in \pi_{n, \infty}^{\theta_{1}}\left(\mathbb{D}_{n}\right), y^{\prime} \in$ $\pi_{m, \infty}^{\theta_{2}}\left(\mathbb{D}_{m}\right), \theta_{1}, \theta_{2} \neq 00$. Therefore, $\mathbf{F}_{(10)}(x \sqcap y)=\emptyset=\mathbf{F}_{(10)}\left(x^{\prime} \sqcap y^{\prime}\right)$.

(ii.2) The coherent subsets $x, y$ do not interpret concurrent processes. Then, by inclusion relation, $x^{\prime}$ and $y^{\prime}$ do not. Therefore, $\mathbf{F}_{(10)}(x \sqcap y)=\mathbf{F}_{(10)}\left(x^{\prime} \sqcap y^{\prime}\right)=\emptyset$.

(ii.3) $\Pi_{\infty, k}^{\vec{b}}(x) \notin \gamma_{k}^{0} \circ \phi_{k}^{1}\left[\mathbb{D}_{k}\right]$. That means, $\Pi_{\infty, k}^{\vec{b}}\left(x^{\prime}\right) \notin \gamma_{k}^{0} \circ \phi_{k}^{1}\left[\mathbb{D}_{k}\right]$. Thus, $\mathbf{F}_{(10)}(x \sqcap$ $y)=\emptyset=\mathbf{F}_{(10)}\left(x^{\prime} \sqcap y^{\prime}\right)$. The same result can be obtained if $\Pi_{\infty, k}(y) \notin \gamma_{k}^{0} \circ \phi_{k}^{1}\left[\mathbb{D}_{k}\right]$. (ii.4) If $x=x^{\prime}=y=y^{\prime}=\emptyset$, it is followed that $\mathbf{F}_{(10)}(x \sqcap y)=\mathbf{F}_{(10)}(x \sqcap y)=\emptyset$.

We prove that $\mathbf{F}_{(10)}(x \sqcap y) \subseteq \mathbf{F}_{(10)}\left(x^{\prime} \sqcap y^{\prime}\right)$.

When $X \sqcap Y$ is a direct subset in $\mathbb{D}_{\infty} \longrightarrow \mathbb{D}_{\infty}^{\rightarrow}$, it happens that $\mathbf{F}_{(10)}(X \sqcap Y)$ is also directed. In fact, let $X, Y \subseteq \mathbb{D}_{\infty}$ be directed subsets in $\mathbb{D}_{\infty}$. Since $\mathbb{D}_{\infty}$ is closed under union of directed sets, $\cup^{\uparrow} X \sqcap \cup^{\uparrow} Y \subseteq \mathbb{D}_{\infty} \longrightarrow \mathbb{D}_{\infty}$. In addition, when $\mathbf{F}_{(10)}$ is a monotonic function it follows that $\mathbf{F}_{(10)}\left(\cup^{\uparrow} X \sqcap \cup^{\uparrow} Y\right)$ is directed.

(i) If $\Pi_{\infty, k}^{\rightarrow}, \pi_{k+1, \infty}^{(00)}$ are continuous functions satisfying the hypotheses, then

$$
\begin{aligned}
\mathbf{F}_{(10)}\left(\cup^{\uparrow} X \sqcap \cup^{\uparrow} Y\right) & =\pi_{k+1, \infty}^{(00)}\left[\Pi_{\infty, k}^{\vec{u}}\left(\cup^{\uparrow} X\right) \cup \Pi_{\infty, k}\left(\cup^{\uparrow} Y\right)\right] \\
& \subseteq \cup^{\uparrow}\left\{\pi_{k, \infty}^{(00)}\left[\Pi_{\infty, k}^{\vec{m}}(y) \cup \Pi_{\infty, k}(x)\right] \mid x \in X, y \in Y\right\} \\
& =\cup^{\uparrow}\left\{\mathbf{F}_{(10)}(x \sqcap y) \mid x \in X, y \in Y\right\} .
\end{aligned}
$$

(ii) Otherwise, if the above hypotheses do not hold, $\mathbf{F}_{(10)}\left(\cup^{\uparrow} X \sqcap \cup^{\uparrow} Y\right)=\emptyset$. Since $\mathbf{F}_{(10)}$ is monotonic, $\mathbf{F}_{(10)}(x \sqcap y)=\emptyset, \forall x \in X, y \in Y$. Therefore $\mathbf{F}_{(10)}\left(\bigcup^{\uparrow} X \sqcap \bigcup^{\uparrow} Y\right)=$ $\bigcup\left\{\mathbf{F}_{(10)}(x \sqcap y) \mid x \in X, y \in Y\right\}$. Thus, we show that $\mathbf{F}_{(10)}$ is continuous.

Consider $x \sqcap y, x^{\prime} \sqcap y^{\prime} \in \mathbb{D}_{\infty} \longrightarrow \mathbb{D}_{\infty}$, such that $(x \sqcap y) \bigcup\left(x^{\prime} \sqcap y^{\prime}\right)=\left(x \cup x^{\prime}\right) \sqcap\left(y \cup y^{\prime}\right) \in$ $\mathbb{D}_{\infty}^{\rightarrow} \prod \mathbb{D}_{\infty}^{\rightarrow}$ and suppose $\Pi_{\infty, k}^{\overrightarrow{ }}, \pi_{k+1, \infty}$ and $\pi_{k+1, \infty}^{(00)}$ are stable functions.

(i) If the hypotheses hold, then

$$
\begin{aligned}
\mathbf{F}_{(10)}\left(x \sqcap y \cap x^{\prime} \sqcap y^{\prime}\right) & =\pi_{k+1, \infty}^{(00)}\left[\Pi_{\infty, k}\left(x \cap x^{\prime}\right) \cup \Pi_{\infty, k}\left(y \cap y^{\prime}\right)\right] \\
& =\pi_{k+1, \infty}^{(00)}\left[\left(\Pi_{\infty, k}(x) \cup \Pi_{\infty, k}(y)\right) \cap\left(\Pi_{\infty, k}\left(x^{\prime}\right) \cup \Pi_{\infty, k}\left(y^{\prime}\right)\right)\right] \\
& =\mathbf{F}_{(10)}(x \sqcap y) \cap \mathbf{F}_{(10)}\left(x^{\prime} \sqcap y^{\prime}\right) .
\end{aligned}
$$

(ii) Otherwise, $\mathbf{F}_{(10)}\left(x \sqcap y \cap x^{\prime} \sqcap y^{\prime}\right)=\mathbf{F}_{(10)}\left((x \cap y) \sqcap\left(x^{\prime} \cap y^{\prime}\right)\right)=\emptyset$ :

(ii.1) If $x \cap x^{\prime}$ and $y \cap y^{\prime}$ do not interpreting concurrent processes, $x, y$ and $x^{\prime}, y^{\prime}$ do not. Then, $\mathbf{F}_{(10)}(x \sqcap y)=\mathbf{F}_{(10)}\left(x^{\prime} \sqcap y^{\prime}\right)=\emptyset$.

(ii.2) If $x \bigcap x^{\prime} \in \pi_{n, \infty}^{\theta_{1}}\left(\mathbb{D}_{n}\right), \quad y \bigcap y^{\prime} \in \pi_{m, \infty}^{\theta_{2}}\left(\mathbb{D}_{m}\right)$, and $\theta_{1}, \theta_{2} \neq 00$, then $x, x^{\prime} \in$ $\pi_{n, \infty}^{\theta_{1}}\left(\mathbb{D}_{n}\right), \quad y, y^{\prime} \in \pi_{m, \infty}^{\theta_{2}}\left(\mathbb{D}_{m}\right)$. Thus, $\mathbf{F}_{(10)}(x \sqcap y)=\emptyset=\mathbf{F}_{(10)}\left(x^{\prime} \sqcap y^{\prime}\right)$. 
(ii.3) If $\Pi_{\infty, k}^{\rightarrow}(x), \notin \gamma_{k}^{0} \circ \phi_{k}^{1}\left[\mathbb{D}_{k}\right]$, it follows that $\mathbf{F}_{(10)}(x \sqcap y)=\emptyset$.

(ii.4) If $x=x^{\prime}=y=y^{\prime}=\emptyset$, by definition, $\mathbf{F}_{(10)}(x \sqcap y)=\mathbf{F}_{(10)}(x \sqcap y)=\emptyset$.

Based on the above cases, $\mathbf{F}_{(10)}$ is an stable function.

Consider $X, Y \subseteq \mathbb{D}_{\infty}$ such that $\forall x, x^{\prime} \in X$ and $\forall y, y^{\prime} \in Y$; we have $x \cup x^{\prime}, y \cup y^{\prime} \in \mathbb{D}_{\infty}$ and $x, x^{\prime} \in \pi_{n, \infty}^{\theta_{1}}\left[\mathbb{D}_{n}\right] \subseteq \pi_{n, \infty}^{\rightarrow}\left[\mathbb{D}_{n}\right], \quad y, y^{\prime} \in \pi_{m, \infty}^{\theta_{2}}\left[\mathbb{D}_{m}\right] \subseteq \pi_{m, \infty}\left[\mathbb{D}_{m}\right]$.

(i)Let be $\Pi_{\infty, k}^{\overrightarrow{,}}, \pi_{\infty, k}^{\vec{a}}$ and $\pi_{k+1, \infty}^{(00)}$ linear functions and suppose that the hypotheses hold. Then,

$$
\begin{aligned}
\mathbf{F}_{(10)}(\cup X \sqcap \cup Y) & =\pi_{k, \infty}^{(00)}\left[\Pi_{\infty, k}(\cup X) \bigcup \Pi_{\infty, k}(\cup Y)\right] \\
& \left.=\bigcup\left\{\pi_{k, \infty}^{(00)}\left[\Pi_{\infty, k}(x) \cup \Pi_{\infty, k}(y)\right] \mid x \in X, y \in Y\right\}\right) \\
& =\bigcup\left\{\mathbf{F}_{(10)}(x \sqcap y) \mid x \in X, y \in Y\right\} .
\end{aligned}
$$

(ii) Otherwise, $\mathbf{F}_{(10)}(\bigcup X \sqcap \bigcup Y)=\emptyset$. Thus, by the monotonicity of function $\mathbf{F}_{(10)}$, proved at the beginning, $\mathbf{F}_{(10)}(x \sqcap y)=\emptyset$, whenever $x \subseteq \bigcup X$ and $y \subseteq \bigcup Y$, i.e., $\mathbf{F}_{(10)}(\bigcup X \sqcap \bigcup Y)=\bigcup\left\{\mathbf{F}_{(10)}(x \sqcap y) \mid x \in X, y \in Y\right\}$.

Based on the above cases, we showed that $\mathbf{F}_{(10)}$ is linear.

\section{The Machine Model and its Computations}

We define the SGM model and its computations based on Scott [9]. Let $\mathcal{S}$ be a set whose elements are represented in the mean value and standard deviation form.

Definition 4.1. The $S G M$ model $\mathcal{M}=\left(\mathcal{M}_{\mathbf{I}}, \mathcal{M}_{\mathbb{D} \rightarrow}, \mathcal{M}_{\mathbb{B}}, \mathcal{M}_{\mathbf{O}}\right)$ is defined as a tuple of functions such that, for input and output values taken in the set $\mathcal{S}$ :

(i) $\mathcal{M}_{\mathbf{I}}: \mathcal{S} \rightarrow \mathbb{S}$ is the input function. When, for instance, $\mathbf{I}=\left\{\mathbf{i}_{00}\right\}$,

$$
\mathcal{M}_{\left\{\mathbf{i}_{00}\right\}}\left(\left(a_{m} ; a_{s}\right)\right)=\{z\}, \quad z(i)= \begin{cases}x_{m} \in \mathbb{I} \mathbb{Q}, & \text { if } i=(0,0,0), \\ x_{s} \in \mathbb{I} \mathbb{Q}, & \text { if } i=(0,0,1), \\ \emptyset, & \text { otherwise. }\end{cases}
$$

where $x_{m}, x_{s} \in \operatorname{tot}(\mathbb{I} \mathbb{Q})$ are the best approximations of $m, s$, defined in $[1]$.

(ii) $\mathcal{M}_{\mathbb{D} \rightarrow \infty}: \mathcal{L}\left(\mathbb{D}_{\infty}\right) \multimap(\mathbb{S} \multimap \mathbb{S})$ is the program interpretation function, such that $\mathcal{M}_{\mathbb{D} \rightarrow \infty}(p)=x$ interprets the program $p$ as the process $x$.

$($ iii $) \mathcal{M}_{\mathbb{B}}: I_{T} \multimap(\mathbb{S} \multimap \mathbb{B})$ is the test interpretation function, such that $\mathcal{M}_{\mathbb{B}}(\mathbf{b})=t$ interprets the symbol $\mathbf{b}$ as the test $t$, as it is defined in $[6]$.

(iv) $\mathcal{M}_{\mathbf{O}}: \mathbb{S} \rightarrow \mathcal{S}$ is the output function. For instance, when $\mathbf{O}=\left\{\mathbf{o}_{i j}\right\}$, then $\mathcal{M}_{\left\{\mathbf{o}_{i j}\right\}}(\mathbf{s})=\left\{(m ; s) \mid z[i, j, 0]=x_{v}, z[i, j, 1]=x_{s} \in \mathbb{I} \mathbb{Q}, s \in \mathbf{s}\right\}$.

The computation of a program $p$ with an input data in results in the production of the output data out $=\mathcal{M}_{\mathbf{O}} \circ \mathcal{M}_{\mathbb{D} \rightarrow \infty}(p) \circ \mathcal{M}_{\mathbf{I}}($ in $)$.

\subsection{Programming stochastic arithmetic in SGM}

A stochastic number stored in the memory of the SGM machine is labelled by a reference position $(l, i) \in \mathrm{R}^{2}$ and a third index indicating its mean value (0) and 
standard deviation (1). In this context, $z[l, i, 0], z[l, i, 1]$ represent, respectively, the mean value and the standard deviation of a stochastic number stored in the reference position $(l, i)$. Some expressions of elementary processes (assignment statements) and their corresponding interpretation in the domain $\mathbb{D}_{\infty}$ are given in Table 1 , where assignment, sum, scalar multiplication, square root, and power operations performed over memory positions are considered. In addition, the abs function returns the absolute value of the real number stored in the memory position.

Table 1: Expressions of elementary processes and their domain interpretations.

\begin{tabular}{|c|c|}
\hline $\mathcal{L}\left(\mathbb{D}_{\infty}^{\rightarrow}\right)$ & $\mathbb{D}_{\infty}$ \\
\hline $\operatorname{ad} \_\mathrm{m}(l, m, n, i, j, k) \equiv(z[l, i, 0]:=z[m, j, 0]+z[n, k, 0])$ & $\left\{a d \_m_{: 00}^{(l i 0)}\right\}$ \\
\hline $\operatorname{ad} \_\mathbf{s}(l, m, n, i, j, k) \equiv\left(z[l, i, 1]:=\operatorname{sqrt}\left(z[m, j, 1]^{2}+z[n, k, 1]^{2}\right)\right)$ & $\left\{a d \_s: 00\right\}$ \\
\hline ad_sy_m $(l, m, n, i, j, k) \equiv(z[l, i, 0]:=\mathbf{0})$ & $\left\{a d \_s y \_m_{: 00}^{(l i 0)}\right\}$ \\
\hline ad_sy_s $(l, m, n, i, j, k) \equiv\left(a d \_s(l, m, n, i, j, k)\right.$ & $\left\{a d \_s y \_s: 00\right\}$ \\
\hline $\operatorname{scm} \_\mathrm{m}(l, i) \equiv(z[l, i, 0]:=z[l, i, 0] * z[l, i, 2])$ & $\left\{s c m_{-} m_{: 00}^{(l i 0)}\right\}$ \\
\hline $\mathbf{s c m} \_\mathbf{s}(l, i) \equiv(z[l, i, 1]:=z[l, i, 1] * a b s(z[s, l, 2]))$ & $\left\{s c m \_s: 00\right\}$ \\
\hline
\end{tabular}

In the following, some compound (non elementary) stochastic arithmetical processes are presented, including the related complexity analysis. Two synchronized processors, identified by the expressions $\mathbf{m}_{(l, i)}$ and $\mathbf{s}_{(l, i)}$, are associated to each memory position $(l, i)$. The elementary processes presented in Table 1 have unitary costs $(c s t=1 u c t)$. For example, $\operatorname{cst}\left(\operatorname{ad} \_\mathrm{m}(l, m, n, i, j, k)\right)=1$, since the action ad_m is allocated to the processor $\mathbf{m}_{(l, i)}$, and $\operatorname{cst}\left(\operatorname{ad}_{-} \mathbf{s}(l, m, n, i, j, k)\right)=1$, since the action ad_s is allocated to the processor $\mathbf{s}_{(l, i)}$.

1. $\operatorname{ad}(l, m, n, i, j, k) \equiv($ ad_m $(l, m, n, i, j, k) \|$ ad_s $(l, m, n, i, j, k))$

This expression denotes the sum of stochastic numbers (see Eq. 2.1), labelled by positions $(m, j)$ and $(n, k)$, with the result placed in the $(l, i)$ position. The sum of the mean value and the standard deviation is performed in parallel at 1uct. Its interpretation $x_{i} \in \mathbb{D}_{\infty}$ can be achieved from the expression:

$x_{i}=\left\{\overline{a d \_m^{(l i 0)}} 10: 00, \overline{a d_{-} s^{(l i 1)}} 10: 00\right\}=F_{(10)}\left(\left\{\overline{a d \_m^{(l i 0)}} 10: 00\right\} \sqcap\left\{\overline{a d \_s^{(l i 1)}} 10: 00\right\}\right)$.

The process ad has unitary cost because both processes ad_m and ad_s, which are performed by different processors in a synchronized way, have unitary costs. Analogous expressions can be obtained using symmetric stochastic numbers:

ad_sy $(l, m, n, i, j, k) \equiv($ ad_sy_m $(l, m, n, i, j, k) \|$ ad_sy_s $(l, m, n, i, j, k))$.

2. $\left(\operatorname{ad} \_r o w(l, m, n, i) \equiv\left(\operatorname{ad}(l, m, n, i, i, i) \| \operatorname{ad} \_\right.\right.$row $(l, m, n, i-1)$

The expression $W=$ ad_row $(l, m, n, 0) \in \mathcal{L}\left(\mathbb{D}_{\infty}\right)$ represents a process that synchronously, performs the addition of the sequence of stochastic numbers stored in the $m$-th and $n$-th rows, and assigns the result to the corresponding positions in 
the $l$-th row, in 1 uct. Its interpretation is obtained by the subset

$$
x=\left\{\overline{a d \_m}^{(l i 0)} 10: 00, \overline{a d \_s}{ }^{(l i 1)} 10: 00\right\}_{i \in \omega} .
$$

Thus, $x$ is the least fixed point of the equation $x_{n+1}=F_{(10)}\left(x_{n} \sqcap x_{0}\right) \in \mathbb{D}_{\infty}$.

Let $P=\left\{\mathbf{m}_{(l, i)}, \mathbf{m}_{(l, i+1)}, \ldots, \mathbf{s}_{(l, i)}, \mathbf{s}_{(l, i+1)}, \ldots\right\}$ be a denumerable set of processors. The process ad_row, which is performed by pairs of processors in the the set $P$ in a synchronized way, has unitary cost.

The corresponding operation performed over $\mathcal{S}_{+}$is given by the expression: ad_sy_row $(l, m, n, i) \equiv($ ad_sy $(l, m, n, i, i, i) \|$ ad_sy_row $(l, m, n, i-1)$.

3. In the sum of stochastic numbers in the first $i$-th rows and $l$-th columns:

$\operatorname{Ad\_ row}(l, 0) \equiv$ skip, Ad_row $(l, i) \equiv\left(\operatorname{ad}(l, l, l, i-1, i, i-1) ; \operatorname{Ad\_ row}(l, i-1)\right)$

$\operatorname{Ad\_ Row}(l, 0) \equiv \mathbf{s k i p}, \operatorname{Ad} \_\operatorname{Row}(l, i) \equiv\left(\operatorname{Ad\_ Row}(l-1, i) ; \operatorname{Ad} \_r o w(l, i)\right)$,

the number of elementary operations either in synchronized or sequential way is the same $(2 i l+l)$, but with different performances, according to the number of processors involved. In the former case, with $i l$ processors, the cost function gives lutc, and, in the later case, using two processors, the complexity is given by a polynomial of third order. The language is flexible enough to allow to substitute the symbol "||" for the symbol ";" whenever one decides to work with either synchronized or sequential computations (in this case, with a least number of processors).

4. Similar analysis can be done for the extended symmetric stochastic addition: $\operatorname{add}(l, m, n, i, j, k) \equiv z(l, i, 1):=\left(\operatorname{sg}(z(m, j, 1)) * z(m, j, 1)^{2}+\operatorname{sg}(z(n, k, 1)) * z(n, k, 1)^{2}\right)$ abs_add $(l, m, n, i, j, k) \equiv(z(l, i, 1):=(\operatorname{abs}(\operatorname{add}(l, m, n, i, j, k))))$ ex_ad $(l, m, n, i, j, k) \equiv z(l, i, 1):=\left(s g\left(\operatorname{abs\_ add}(l, m, n, i, j, k)\right) * \operatorname{sqrt}\left(\operatorname{abs\_ add}(l, m, n, i, j, k)\right)\right)$ and for the scalar multiplication over the stochastic numbers. In the last case, $\mathrm{P}$ is a recursive process performing the scalar multiplication over the stochastic numbers placed in the first $l$-th columns of the $i$-th row, simultaneously:

$P=z(l, i, 2):=\alpha ; \mathbf{s c m} \_r o w(l, i):=\left(\mathbf{s c m} \_\mathrm{m}(l, i) \| \mathbf{s c m \_ s}(l, i)\right) \| \operatorname{scm} \_r o w(l, i-1)$.

\section{Conclusion and further works}

The ordered structure of the SGM provides domain theoretic semantics for compound (non elementary) sequential algorithms with operations of the Stochastic Arithmetic. In this work, we take use of the linear operator $F_{(10)}$ to obtain the related compound parallel processes performed over array structures of stochastic numbers, in a synchronous approach. We introduced a connection between syntax and domain-theoretic semantics of parallel stochastic algorithms related to the stochastic arithmetic operation. The complexity analysis of sample operations and algorithms made clear the adequacy of the chosen representation of stochastic numbers in the memory of the SGM, and the performance benefit of the parallel structuring of stochastic arithmetic programs.

Resumo. Apresenta-se a versão estocástica da Máquina Geométrica, capaz de interpretar computações sequenciais, paralelas (síncronas) e não determinísticas, 
considerando uma memória compartilhada (posssivelmente infinita). A linguagem de programação $\mathcal{L}\left(\mathbb{D}_{\infty}\right)$, induzida pelo espaço coerente de processos $\mathbb{D}_{\infty}$, pode ser aplicada sobre produtos sequenciais e paralelos, para prover definições recursivas de processos estocásticos e uma semântica denotacional para a Aritmética Estocástica. São analisadas a recursão espacial (ou ordinal) relacionada com a modelagem espacial da memória, e a recursão temporal (ou estrutural) obtida pela relação de inclusão modelando objetos parciais na estrutura do modelo. O estudo da complexidade de algumas operações e algoritmos aritméticos estocásticos mostrou a adequação da representação escolhida para os números estocásticos, assim como as vantagens da estruturação paralela de programas de aritmética estocástica.

\section{References}

[1] G.P. Dimuro, A.C.R. Costa and D.M. Claudio, A Coherence Space of Rational Intervals for a Construction of IR, Reliable Computing, 6, No. 2 (2000), 139178.

[2] J. -Y. Girard, Linear Logic, Theoretical Computer Science, 1 (1987), 187-212.

[3] S. Markov, On the Algebraic Properties of Intervals and Some Applications, Reliable Computing, 7, No. 2 (2001), 113-127.

[4] S. Markov and R. Alt, Stochastic Arithmetic: Addition and Multiplication by Scalars, Applied and Numerical Mathematics, 50 (2004), 475-488.

[5] R.E. Moore, "Methods and Applications of Interval Analysis", SIAM, 1979.

[6] R.H.S. Reiser, A.C.R. Costa and G.P. Dimuro, First steps in the construction of the Geometric Machine, em "Seleta do XXIV CNMAC" (E.X.L. de Andrade, J.M. Balthazar, S.M. Gomes, G.N. Silva and A. Sri Ranga, eds.), Tendências em Matemática Aplicada e Computacional, Vol. 3, pp. 183-192, SBMAC, 2002.

[7] R.H.S. Reiser, A.C.R. Costa and G.P. Dimuro, A programming language for the Interval Geometric Machine, Electronic Notes in Theoretical Computer Science, 84 (2003), 1-12.

[8] R.H.S. Reiser, G. P. Dimuro and AC. R. Costa, The Interval Geometric Machine Model, Numerical Algorithms, 37, No. 4 (2004), 357-366.

[9] D. Scott, Some definitional suggestions for automata theory, Journal of Computer and System Sciences, 1, No. 1 (1967), 187-212.

[10] V. Stoltenberg-Hansen, I. Lindström and E. R. Griffor, "Mathematical Theory of Domains", Cambridge University Press, Cambridge, 1994. 